\title{
A Case-Based Study of Students' Visuohaptic Experiences of Electric Fields around Molecules: Shaping the Development of Virtual Nanoscience Learning Environments
}

\author{
Gunnar E. Höst, Konrad J. Schönborn, and Karljohan E. Lundin Palmerius \\ Department of Science and Technology, Linköping University, 60174 Norrköping, Sweden \\ Correspondence should be addressed to Konrad J. Schönborn; konsc@itn.liu.se
}

Received 5 October 2012; Accepted 13 January 2013

Academic Editor: Zubair Amin

Copyright (c) 2013 Gunnar E. Höst et al. This is an open access article distributed under the Creative Commons Attribution License, which permits unrestricted use, distribution, and reproduction in any medium, provided the original work is properly cited.

Recent educational research has suggested that immersive multisensory virtual environments offer learners unique and exciting knowledge-building opportunities for the construction of scientific knowledge. This paper delivers a case-based study of students' immersive interaction with electric fields around molecules in a multisensory visuohaptic virtual environment. The virtual architecture presented here also has conceptual connections to the flourishing quest in contemporary literature for the pressing need to communicate nanoscientific ideas to learners. Five upper secondary school students' prior conceptual understanding of electric fields and their application of this knowledge to molecular contexts, were probed prior to exposure to the virtual model. Subsequently, four students interacted with the visuohaptic model while performing think-aloud tasks. An inductive and heuristic treatment of videotaped verbal and behavioural data revealed distinct interrelationships between students' interactive strategies implemented when executing tasks in the virtual system and the nature of their conceptual knowledge deployed. The obtained qualitative case study evidence could serve as an empirical basis for informing the rendering and communication of overarching nanoscale ideas. At the time of composing this paper for publication in the current journal, the research findings of this study have been put into motion in informing a broader project goal of developing educational virtual environments for depicting nanophenomena.

\section{Introduction}

1.1. Immersive Visuohaptic Virtual Environments for Learning Much literature in educational research concerned with the learning of science has put forward the premise that modern multimodal and immersive virtual learning platforms have the potential to offer conceptually rich and exciting learning opportunities (e.g., [1]). In this regard, building multisensory environments that simultaneously exploit visual and haptic sensory perception can provide learners with the opportunity to experience and interact with virtual worlds specifically designed to depict natural phenomena. Through such technological development, other than perceiving information visually and verbally, modern visuohaptic virtual environments also engage a user's tactile sensory pathway. Herein, engagement of this sensory channel is concerned with haptics and refers to the perception of force stimuli such as the texture, hardness, and shape of virtual objects [2]. An example of exploiting haptic perception for learning science has been demonstrated by Reiner [3] who showed that tactile feedback can help students develop accurate concepts of force fields.

Although visuohaptic virtual environments could certainly be conducive for learning, to date, little empirical educational research has been directed to these platforms, and there remain gaps in the findings about how existing results concerning immersive environments that integrate visual information with haptic feedback can be used as a basis for informing the building of innovative technologies for enhancing the formal learning of science [4].

From a cognitive point of view, and with respect to the pedagogic merits of visual only versus visuohaptic virtual learning environments, educational research conducted by 
our group concerned with learning about complex molecular phenomena (e.g., [5]) has shown that from an informationprocessing point of view, coordinating visual and haptic perception may place less strain on visual working memory because required processing can get "offloaded" onto the tactile pathway. In addition, from an embodied cognition point of view [6], visuohaptic sensorimotor experiences in a virtual macroworld could provide active entry points for building conceptual knowledge about otherwise unobservable submicroscopic phenomena. Although these results have educational implications for the utilisation of visuohaptic virtual reality technologies, Dede [7] suggests that much more research needs to be directed towards aligning the intended instructional design behind such innovation with the nature of the virtual immersion offered by the system, as well as considering learners' strengths and preferences in using different virtual systems. At the same time, much more educational research is needed to uncover the cognitive underpinnings in any differences between "no haptics" (e.g., visual only) and "haptics" (visuohaptic) virtual environments and to investigte how the nature of the interactive learning context in relation to what concepts are targeted for learning impacts these dimensions (e.g., $[8,9]$ ).

1.2. Scientific Concepts Related to the Electrostatic Properties of Molecules. Electric fields exist around any stationary charged objects, exerting a force on any charged particle within the field. Even though the idea of electric field serves as a crucial concept for pupils to grasp at the school level, multiple studies in the educational research literature suggest that students often find the concept extremely challenging. For instance, students sometimes struggle to connect electric field properties with the corresponding force exerted on a charged particle and often reveal a "matter-based" conceptualisation of electric field lines (e.g., $[10,11])$. In addition, Törnkvist et al. [12] have demonstrated that pupils regularly tend to conceptualise electric field lines as separate entities, rather than in light of complementary electric field vectors. Furthermore, the concept of electric field is pivotal to the understanding of molecular interactions at large, given the electrostatic nature of essentially all intermolecular forces. By the same token, electrostatic properties of molecules are also linked to chemical polarity and molecular shape concepts. In this regard, any molecular charge distribution, such as that associated with monopoles (e.g., $\mathrm{K}^{+}, \mathrm{Cl}^{-}$), dipoles (e.g., $\mathrm{H}_{2} \mathrm{O}$ ), and quadrupoles (e.g., $\mathrm{CO}_{2}$ ), all exhibit a corresponding electric field. Therefore, even molecules that exhibit a dipole moment (i.e., "polar") as well as many of those that do not (i.e., "nonpolar") always give rise to an electric field.

Many investigations in the literature have shown that interactive learning environments may promote students' adjustment of erroneous existing conceptual understandings toward more scientifically accurate conceptions (e.g., [13]). With respect to virtual environments that communicate concepts related to electric fields, Dede et al. [14] found improvements in conceptual understanding among students after their interaction with a virtual model that visually compared electric force with potential by depicting field lines and charge traces associated with dipoles. Earlier research by these workers [15] involved the development of a multimodal platform that integrates visual, auditory, and haptic feedback with the objective of improving learners' understanding of demanding, yet pivotal, scientific concepts such as electrostatics. Aspects of their results delivered upon human interaction with the system that incorporated perceiving haptic cues showed that students were more attentive, felt that the virtual environment made their understanding about physics concepts more meaningful, and outperformed other groups with respect to core concepts such as acceleration. Lastly, among other haptic features, Křenek [16] has developed a visuohaptic model to represent aspects of electron density and corresponding rendered haptic force fields.

1.3. Technical and Interactive Aspects of the Presented Visuohaptic Virtual Environment. The haptic virtual model presented in the current study builds on previous work by Palmerius et al. [17] on scientific visualisation with haptic feedback. A set of algorithms developed for the perceptualisation of volumetric data allow for the effective use of multisensory interaction and integration of stimuli. Components of this work can be combined in various ways to build different virtual models. In the currently employed model, a molecule is visually rendered using a "ball-and-stick" representation. In addition, a semitransparent visual rendering of the van der Waals surface of the molecule can be activated by the user. The model is presented to the user in a semi-immersive mirrored display (Figures 1 and 3), where 3D stereorendered graphics are reflected and colocated with a haptic device (a Desktop PHANToM from Sensable). The graphic and haptic rendering are calibrated and coregistered so that the PHANToM probe is used in $3 \mathrm{D}$ to explore the molecule's electrostatic potential at the position where the probe point is also seen (Figure 1). A 3D mouse is used to enable the rotation and alignment of the molecule using one's other hand. The electric field surrounding the molecule is calculated from a point charge representation of the molecule and presented visually by generating "stream-tubes" via pushing a button on the haptic device and haptically through the haptic interface (Figure 1).

Two different haptic modes are used for interacting with the electric field. In a force mode, the probe is exposed to a force aligned with the field, as if it was a positive charge. In this mode, the user feels the probe being pulled along the field towards negatively charged molecular regions and pushed away from positive regions. In a force-and-follow mode, the previously described effect is combined with force feedback that guides the probe in following the field direction. The added effect provides a resistance when trying to move the probe across the field although it is weak enough to allow for free exploration. The combined effect is that the user feels that the probe is pulled along the field towards the charge while also feeling the shape of the electric field, which can be interactively explored.

Given that there has been surprisingly little attention given to whether and how learners merge their "chemical" understanding about molecules with their "physical" understanding of electric fields, we aimed to exploit the interaction 


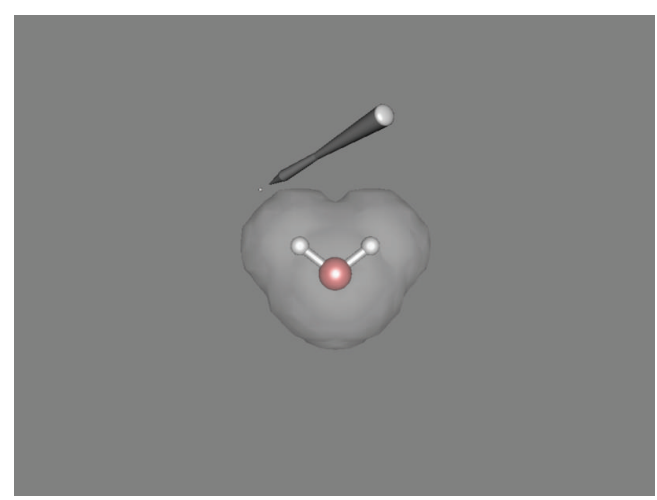

(a)

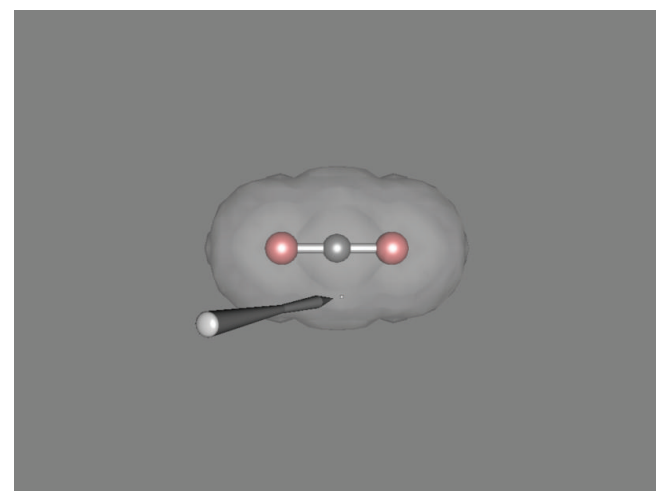

(c)

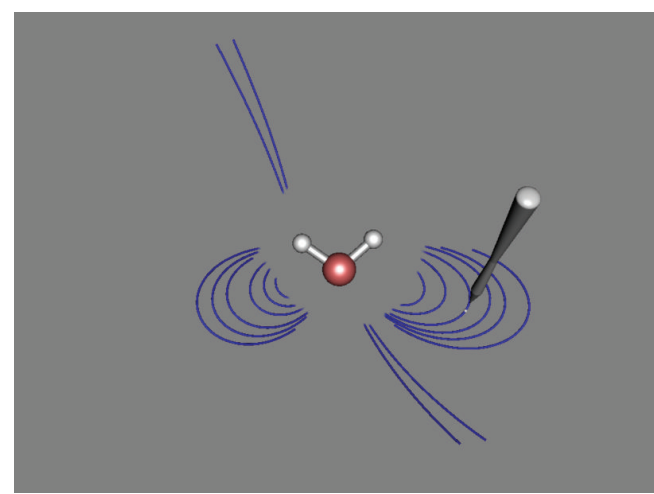

(b)

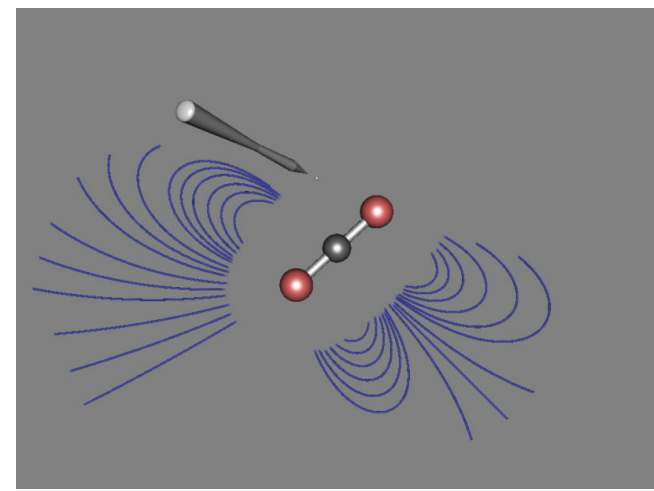

(d)

FiguRE 1: A panel of four screenshots from the virtual system displaying an $\mathrm{H}_{2} \mathrm{O}$ molecule with a van der Waals surface (a), an $\mathrm{H}_{2} \mathrm{O}$ molecule with visual field lines (b), and a corresponding $\mathrm{CO}_{2}$ molecule displayed in the same modes (c) and (d), all in a "ball-and-stick" representation. While interacting with the system, the viewer receives a 3D stereoscopic view of the molecules and can also rotate them. The stylus that represents the handle of the haptic device is also shown with the probe (small white sphere).

offered by the present virtual model to investigate such a premise.

\subsection{Connections between the Virtual Model and Nanoscale} Ideas. In light of the presented virtual learning environment above, there has been a flourishing interest in recent educational research for communicating meaningful knowledge to students about science and technology at the nanoscale. The nanoscale comprises processes and objects of which at least one structural dimension is in the range of 1-100 nanometres. Put differently, the nanoscale is the level of scale that is a billionth (0.000000001) smaller than what we typically encounter during our everyday experience. The current interest in the education literature about communicating nanorelated ideas to students (e.g., [18]) is born primarily out of the notion that an impending "nanorevolution" is presenting a pressing need to provide learners with conceptual knowledge underlying the basic ideas of nanoscience.

With the above proposition in mind, we postulated that one way to deliver communication of abstract and otherwise unobservable nanoscale phenomena is to construct $3 \mathrm{D}$ immersive virtual architectures wherein learners' multisensory interactive experiences with such systems can serve as the cognitive access points for constructing nanorelated conceptual understanding. The thinking behind this hypothesis is that given the fact that the model allows for the opportunity to actually feel virtual objects, such haptic perception could stimulate learners to integrate the offered sensorimotor experiences into their construction of the intended underlying scientific knowledge. On this score, this line of reasoning supports an embodied cognition standpoint (e.g., [6]), which offers the position that bodily experiences are a key aspect of cognition, and constructing scientific understanding is deeply interlaced with sensorimotor interaction in the world. Mapped to a virtual world then, experiencing a visuohaptic representation of nanoproperties becomes one pathway for interpreting and reasoning about aperceptual nanostructures in the form of multisensory macroscopic analogies (cf. [19]).

In this regard, examples of nanoconcepts and associated nanoproperties that would be applicable to the presented model (Figures 1, 3, and 4) include parallels with recent literature from Stevens et al. [20], Hingant and Albe [21], and Batt et al. [22] and could be related to overarching nanoprinciples related to forces and interactions between nanoobjects, the shape of nanoobjects, and aspects of relative volume and scale of nanoobjects. 


\section{Research Aim}

Given the motivation above, a broad project vision has recently been put into motion by the granting of research funding to develop and investigate virtual nanotechnology environments for communicating and learning about nanophenomena. As part of responding to this overall research agenda, we have employed initial case-based results from the model presented here (Figures 1, 3, and 4) to inform our future conceptualisation and development of multisensory interactive virtual environments in a nano-education context. In this paper, we present explorative case-based results in the context of students' interpretation of electric fields around molecules, and then use these findings to predict how they could serve as a research basis from which to develop environments for learning nanoscientific concepts. Specifically, the study posed the following research questions.

(1) What are students' "baseline" conceptions about electric fields when applied to a molecular context, before any exposure to a virtual haptic model that renders the phenomenon?

(2) How does interacting with the multisensory visuohaptic model influence students' conceptual understanding of electric fields around molecules?

(3) How can the findings be used as an empirical basis for informing the development of educational virtual environments for learning about nanoscientific phenomena?

We are presently attempting to apply the research findings reported in this paper as one foundation for informing the development of multisensory virtual environments that can be used as educational platforms for communicating, and learning about, nanorelated concepts.

\section{Methods}

3.1. A Case-Based Approach for Responding to the Research Questions. The research design reported here employed a case-based approach to respond to the research questions. In this regard, we followed Eisenhardt's [23] premise of a case study approach as "a research strategy which focuses on the dynamics present within single settings" (page 534). Such approaches are often exploratory and employed to provide descriptions, uncover theory, and/or inform future research prospects that emerge from the case-based data [24]. Therefore, a case study approach is often an embedded methodology that involves numerous levels of analysis, often within the same study, to generate descriptive accounts of learning processes in relation to a specific context (e.g., [25]). Case-based approaches are viewed as providing educational researchers with insight into students' processes of understanding and knowledge acquisition because the method is aimed at extensive interpretative analyses of only a single or a few case(s) representative of a particular context $[25,26]$.

Our case-based approach was characterised by three components as defined by Merriam [27]. Firstly, our approach was particularistic in that it focused on investigating a specific and bounded situation or phenomenon. Secondly, our approach pursued a rich description of the entity being investigated, which often entails a qualitatively-dominated approach to data treatment. Thirdly, our approach was heuristic since we wished to expand an existing understanding of the phenomenon being studied by providing a new awareness about a particular learning context. In the latter respect, one clear objective of the method was to use the case-based approach as an empirical basis for informing the design of virtual environments for learning about and communicating nanoscientific concepts. In this sense, any patterns of interaction revealed by the case-based approach could inform and provide insight into what features of the environment have to be considered if we are to foster knowledge building in a nanotechnology learning context.

3.2. Students and Data-Collection Instruments. By employing the case-based approach described above, data were gathered through an explorative paradigm (e.g., [28]). Five 11th and 12 th grade volunteers (designated $S 1$ through $S 5$ in this paper) enrolled in a natural science program at a Swedish secondary school participated in the study. Necessary ethical requirements stipulated by the Swedish authorities for obtaining research data from the participants were strictly adhered to. To investigate students' conceptual knowledge before exposure to the virtual environment, participants provided written responses and produced student-generated diagrams (SGDs) in response to six written probes concerned with electric fields and electrostatic forces.

After responding to the written test, four of the five students (S1-S4) partook in semistructured interviews in conjunction with interacting with the virtual model. This process entailed completing a series of tasks while the various haptic and visual modes of the model were activated. Two example tasks, where students used the force and force-and-follow haptic modes consisted of the following.

\begin{abstract}
On the screen there is an $\mathrm{H}_{2} \mathrm{O}$ molecule depicted in $3 \mathrm{D}$ ball-and-stick format. The $\mathrm{H}_{2} \mathrm{O}$ molecule has a region that is partially positively charged, and a region that is partially negatively charged. Consider that the small white ball at the tip of the pen is a positive point charge. Interact with the haptic model to describe and deduce the position of the positively and negatively charged regions of the molecule.
\end{abstract}

A $\mathrm{CO}_{2}$ molecule is shown on the screen. Imagine that you are positioned at the tip of the pen. Generate field lines around the molecule [by pushing the button on the stylus]. Select one [generated] stream tube and use the haptic device to follow that trajectory. Fully explain the relationship between what you see and what you feel and how this is related to the properties of the molecule.

The data-collection procedure placed much emphasis on stimulating students to "think-aloud" while interacting with the virtual model (e.g., [29]). All four interviews were video 
recorded and fully transcribed. A dual monitor that captured students' movement of the stylus and virtual molecule on the screen was also videotaped (see Figures 3 and 4).

3.3. Data Analysis. The dataset analysed in this study consisted of written responses, SGDs, and videos of students' interaction with the virtual model. Data analysis was of a qualitative disposition. We first analysed students' written answers and SGDs to the probes as a baseline measure of the concepts that students brought to the model. Secondly, we analysed the interactive think-aloud sessions inductively, while also considering the nature of students' prior knowledge as revealed by the former analysis. Overall, we followed an interpretative, heuristic and iterative approach to the analysis of the data, which is an often employed method in empirical studies that investigate the conceptual learning merits of novel educational innovations (e.g., [30, 31]). As part of this methodological paradigm, we had the objective of exploring students' interaction with the virtual environment by allowing any patters of observation to emerge naturally, rather than being predisposed $[32,33]$.

Each of the four students that participated in the thinkaloud real-time interactions with the model was treated as separate "case studies," and the data were analysed at two levels. One level comprised of each individual student's interaction with the system, while the other concerned all four student cases as a collective. Here, within-case analyses explored each student's interaction with the model, while a cross-case analysis considered the group (e.g., [34]). In analysing this data, we were concerned with discovering the processing and meanings that each individual case employed or created in each particular interactive (learning) context (e.g., [35]), as well as uncovering patterns across the group as a whole. In line with other case-based approaches in the literature (e.g., $[25,36])$, doing so entails a comprehensive analysis of a few cases representative of learning processes in relation to a particular context. This involved the analysis of rich verbal and behavioural output that participants exposed during the tasks (e.g., [36]). Herein, such an exploratory case-based approach was adopted as a means for providing descriptions underlying students' interactions and for informing future research prospects that could potentially emerge from the case-based data (e.g., [24]).

Specifically, our analytical approach was similar to that conducted by Wu et al. [34], where we wished to uncover any patterns of interaction while students used the model, as well as observe how students linked their conceptual knowledge (obtained from the pretest written probes in our study) to the information embedded in the virtual representations. We did so by analysing the behavioural interactions captured in each video interview in conjunction with the verbal transcripts to develop themes and patterns of interactive behaviours and strategies demonstrated by the students and the related conceptual dimensions of students' engagement with the model.

The practical data analysis procedure was implemented as follows. Transcripts from the think-aloud sessions were read carefully, and any interpreted instances of verbal connections to either interactive experiences or conceptual knowledge were marked. Students' interactive behaviour as captured by the video recordings also guided the interpretation of the verbal information. For example, if a student referred to "a force pulling towards this negative atom here," the visual information in the video could be used to interpret whether the verbal statement reflected a scientifically correct assignment of the sign of the partial charge on the particular atom close to the probe point. As the analytical procedure was iterated, a more refined structural description of the data emerged naturally in the form of four distinct themes. The themes describe differences between students' conceptual engagement and interactive behaviour, respectively, as well as interrelations between these two dimensions (see Table 1).

Although the data treated in the study was limited to five student cases, our objective was on a rich and descriptive exploration of individual interaction with the model, rather than on inferring generalisations about the findings. In this manner, the focus was on a heuristic case-based analysis of the data rather than on pursuing quantitative inference.

\section{Findings and Discussion}

The findings from this study are presented as follows. Firstly, we present results from written responses related to students' conceptions about electric fields, and how these are applied to a molecular context. Secondly, we articulate the overall themes that emerged from qualitative analysis of each of the student cases during the think-aloud interactions with the virtual model. The four overall themes that emerged from an inductive treatment of the data are presented in Table 1. Thirdly, we unpack each theme in relation to the presentation of anchor datum examples. An anchor example (cf. [37]) is described as a datum which, we feel effectively, conveys the nature of a particular theme (Table 1). As an implication of the case-based analysis, we then proceed to map the obtained findings from the case studies to a consideration of the development of an educational virtual environment for communicating and learning about nanophenomena in Section 5.

4.1. Students' Conceptions about Electric Fields and How They Are Applied to Molecules. Analysis of the written data revealed that only a single student (1/5) was able to meaningfully associate field lines to the charge distribution in a water molecule (Figure 2(a)). Although analysis showed that students conveyed scientifically accurate understandings of an electric field in terms of a physics context, they failed to apply this concept to a molecular context. Moreover, analysis also indicated that the presence of an electric field was strongly linked to the idea of molecular polarity, where students erroneously argued that no electric field is associated with nonpolar molecules (e.g., $\mathrm{CO}_{2}$, Figure 2(b)).

Inappropriate mapping of the absence of an electric field around a molecule onto the idea of chemical nonpolarity may be due to statements in learning resources suggesting that molecules are nonpolar when molecular symmetry is such that any partial charges "cancel" each other (e.g., [38, page 30]) instead of making it clear that there is no overall dipole due to the symmetry of the molecule. 
TABLE 1: Intersections of students' deployment of conceptual knowledge (construction or adjustment of concepts/engagement of existing concepts) with emergent interactive strategies (confirmatory/exploratory) observed in think-aloud tasks with a visuohaptic virtual model of molecular electric fields.

\begin{tabular}{lll}
\hline & Confirmatory & Exploratory \\
\hline \multirow{2}{*}{ Construction or adjustment of concepts } & $\begin{array}{l}\text { Validating newly constructed or adjusted concepts } \\
\text { by comparing perceptions within the system }\end{array}$ & $\begin{array}{l}\text { Constructing or adjusting concepts based } \\
\text { on perception }\end{array}$ \\
& $\begin{array}{l}\text { Verifying existing concepts by comparing } \\
\text { Engagement of existing concepts }\end{array}$ & $\begin{array}{l}\text { Interpreting perceptions in terms of } \\
\text { perceptions within the system to those expected }\end{array}$ \\
\hline
\end{tabular}

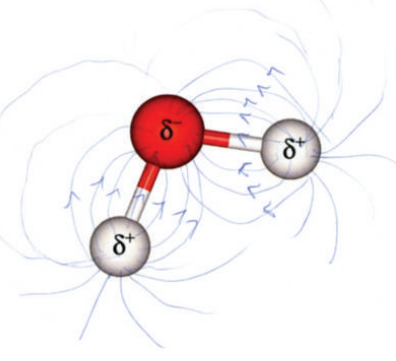

(a)

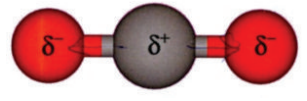

(b)
FIGURE 2: Two SGDs obtained from the written data. (a) accurately represents the overall shape of the electric field around an $\mathrm{H}_{2} \mathrm{O}$ molecule. However, the electric field that would be exhibited by the $\mathrm{CO}_{2}$ molecular charge distribution is not depicted in diagram (b). The two arrows inserted erroneously indicate that the charges pointing in opposite directions "cancel" each other out so that no electric field arises.

4.2. Intersections of Students' Deployment of Conceptual Knowledge and Emergent Strategies during Multisensory Interaction with the Visuohaptic Model. The case-based analysis of students' verbalizations and accompanying behaviours revealed two main dimensions associated with students' execution of the tasks in the virtual environment: students' deployment of conceptual knowledge and students' accompanying interactive strategies (Table 1). The deployment of conceptual knowledge consisted of either engaging existing concepts or actively constructing or adjusting concepts. Here, engagement of existing concepts refers to instances where concepts are not necessarily changed, for example, in the process of retrieving conceptual knowledge from long term into working memory (e.g., [39]). Although the construction or adjustment of concepts will certainly involve the engagement of existing conceptual knowledge, the difference between the two categories is that in engagement there is no change to the nature of the existing concepts, while construction or adjustment involves an active conceptual change that is qualitatively evidenced in the collected verbal and behavioural data (e.g., [40]). Deployment of conceptual knowledge often occurs while a learner either manifested a distinctly confirmatory or exploratory strategy to interact with the virtual system. The junctions of this $2 \times 2$ interrelation are revealed as the emergent themes in the data that are unpacked in the sections below.
4.2.1. Case-Based Evidence for Constructing or Adjusting Concepts Based on Perception. Analysis revealed that students sometimes processed their interactive experiences in the construction of new conceptual understanding. In many cases, students expanded their knowledge of the electric field around the molecule that they investigated by interpreting their interactive experience. In some cases, the construction of new conceptual knowledge could also be described as an indication of conceptual change. In particular, in some cases, a transformation of students' understanding took place as the student made sense of the haptic and visual features of the model. For example, in the following excerpt, student S4 was shown to deliver the following verbal output while the haptic force-and-follow mode was activated:

\section{S4: It [the existence of an electric field] had noth- ing to do with the fact that it [the $\mathrm{H}_{2} \mathrm{O}$ molecule] was polar. \\ I: Did you realise something there? \\ S4: Yes I think so, since the oxygen is negative on both sides [the ends of the $\mathrm{CO}_{2}$ molecule], delta negative or the net charge is negative and the one [atom] in the middle, the carbon, it is positively charged, and the pen [the stylus] is still attracted to the oxygen atoms because they are negative.}

\section{$(26: 25-27: 25$ min. $)$}

Earlier interaction with the virtual $\mathrm{H}_{2} \mathrm{O}$ molecule revealed that $\mathrm{S} 4$ was of the strong opinion that only polar molecules are associated with electric fields. However, when experiencing a haptic representation of the electric field surrounding $\mathrm{CO}_{2}, \mathrm{~S} 4$ appeared to come to the realisation that nonpolarity does not necessarily indicate the absence of an electric field. This case serves as a clear situation where the interactive experience actively challenged the student's existing alternative conception and acted as a platform for inducing an adjustment in the student's conceptual structure, which is notoriously difficult to achieve through traditional means (e.g., [14]). Furthermore, the interaction in this case supports Reiner's [3] observation that a tactile experience "acts as a gate to retrieve tacit knowledge and recruit it for learning” (page 51).

In another example, student S1 exposed the following exchange with the interviewer concerning recently generated visual field lines (stream tubes):

S1:.. you could say that the lines show how it [a charged probe] is affected. 


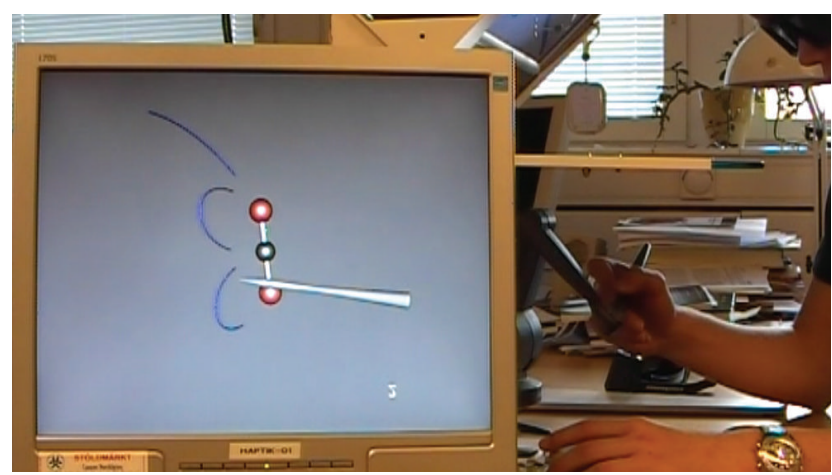

Figure 3: Video screenshot from the think-aloud session with S1. A $\mathrm{CO}_{2}$ molecule surrounded by three field lines (blue) is shown. S1 probes a recently introduced field line using the haptic device (right hand). Also shown are the stereoglasses (top right) and the 3D mouse (left hand) that the student uses to manipulate the molecule during interaction within the virtual environment.

I: Yes, okay, and that you happened to come to [the position the stylus is at] that particular line, what do you think that depends on, or are there more [lines], how many lines are there?

S1: There should be, there really should be an arbitrary number... because no matter where I end up in this space [volume surrounding molecule] I should be affected [by the field], it should not be that if I come in [position the stylus] between two lines then I will not be affected.

\section{$(32: 48-33: 30 \mathrm{~min}$.}

The datum above suggests that the student's understanding of electric field lines is that they are only a representation of the direction of force that a charged positive particle would experience in that vicinity. In addition, the statement that electric fields could be represented by an infinite number of possible field lines also demonstrates a meaningful assimilation of new conceptual knowledge. This was further supported by S1 who revealed after the interview that he did not have this knowledge prior to being exposed to the model. In this instance, the model has provided the student with unique insight into the nature of an electric field that would otherwise be difficult to attain (e.g., [14]).

\subsubsection{Case-Based Evidence for Validating Newly Constructed} or Adjusted Concepts by Comparing Perceptions within the System. This theme was characterised by statements where students generated predictions based on conceptual knowledge gained from interpreting their interactive experience, followed by testing their formulated predictions within the virtual environment. This active learning process exploits the interactivity of the system and allows the students to experiment within the immersive space. For example, this interaction often took the form of a confirmation that the electric field is symmetrical along an axis of symmetry of the molecule. In the following quotation, student S1 predicts the shape of the field lines in areas where no lines have yet been visually placed. Following this event, S1 then goes on to investigate and confirm the prediction as represented in the exchange below:

\section{I: Okay, and if you would choose another position to place one [field line] at? \\ S1: Here, one [field line] goes between these two [atoms], let us see, and there should be a similar [field] over here [probes field in a symmetric posi- tion, followed by placing field line, see Figure 3]
I: Was it like you expected?
S1: Yes. (26:48-27:09 min.)

4.2.3. Case-Based Evidence for Interpreting Perceptions in Terms of Existing Concepts. This theme in the data described instances where students interpreted their experiences of interacting with the virtual environment in terms of their existing concepts. In the following excerpt, student S3 relates the experience of "following along" a field line using the force-and-follow haptic mode to existing prior knowledge of attraction between opposite charges:

\section{I: What do you think it is that makes it follow the line, then? \\ S3: Well, the oxygen is negatively charged and the ball (probe point) is positively charged so they will be drawn towards each other.}

\section{$(27: 31-27: 48$ min. $)$}

In another example, student S4 identifies the visual field lines as a representation of an electric field by linking them to a prior understanding of the structure of magnetic fields:

S4: Oh, right, it is an electric field.

I: How did you see that?

S4: Because they... Let's see [places more field lines]. Yes, they look so much like magnetic fields, or electric-that is... Since there are charges on the molecule, or they have different poles, an electric field will arise, and therefore I assume it is an electric field [...]

\section{$(17: 54-18: 28 \min$.}

As described in Section 4.2.1, student S4 initially assumed that only polar molecules are associated with electric fields. This statement indicates that this exposed alternative conception may be associated with confusion between the nature of magnetic fields and electric fields. Later in the interview, student S4 confirms this assertion by pointing out that most students have had experiences of using magnets in school, and that these magnets always have two poles. Further support for this notion was yielded by other students who also compared electric fields with magnetic fields, using the earth's magnetic field as an example. 


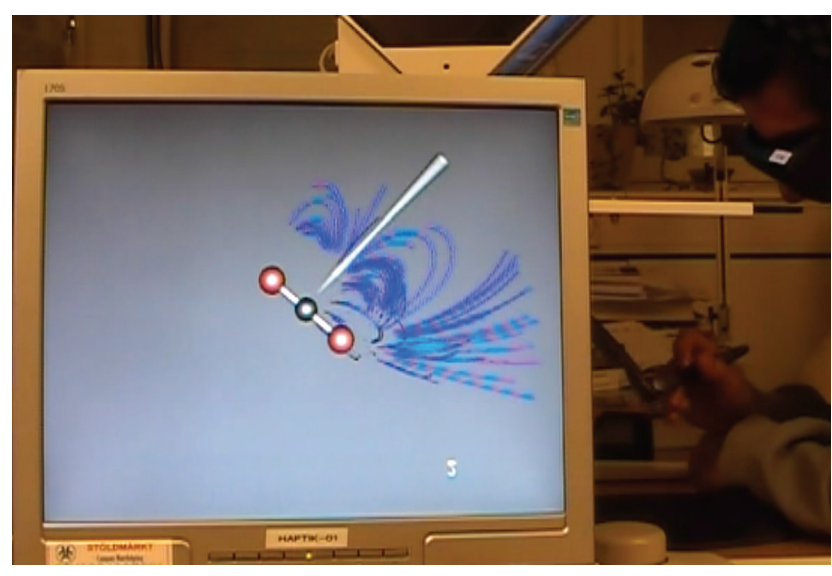

FIGURE 4: Student S2 uses the haptic device to probe the centre of the carbon atom in $\mathrm{CO}_{2}$ during interaction with the system. In contrast with the verbal output obtained from S2, the visuohaptic model does not in fact provide any haptic feedback in the region probed by the student in this specific episode.

4.2.4. Case-Based Evidence for Verifying Existing Concepts by Comparing Perceptions within the System to those Expected. Instances of this category were observed for student S2, who seemed to use existing conceptual understanding as a guide to direct perceptual attention when interacting with the model. In effect, this strategy involves the student forming an expectation of what the perceptual experience will be like, and then using that experience as a lens for filtering the actual experienced perceptions. It should be noted that it is difficult to verify the degree to which expectations modulate students' haptic experiences in other cases. However, in the following two excerpts, student $\mathrm{S} 2$ specifically describes differences in the haptic experience that were not actually outputted by the model and that none of the other students remarked upon.

In the first example, the student (S2) compared the forces around a presently rendered $\mathrm{CO}_{2}$ molecule under examination with the $\mathrm{H}_{2} \mathrm{O}$ molecule investigated during the preceding task:

\section{S2: I do not feel at all as much resistance and it is probably because [...] carbon dioxide is a gas, a volatile gas so it cannot have a strong electric force around it, I think, because that is what makes it a gas in its natural form.}

\section{$(30: 23-31: 21 \mathrm{~min}$.}

Based on a previous understanding of the properties of molecules that form gases, S2 claims that the field is weaker around the $\mathrm{CO}_{2}$ molecule. However, the field around this molecule actually gives rise to similar forces as in an $\mathrm{H}_{2} \mathrm{O}$ molecule. In an event that follows later, the following quotation captures S2 reflecting upon the haptic experience when probing the centre of the carbon and oxygen atoms via this erroneously expected perception (Figure 4):

S2: Since it is a volatile gas there cannot be as strong an electric field here [...] I feel a very

\begin{abstract}
insignificant resistance at the carbon atom [probes carbon atom centre, see Figure 4] compared to the oxygen atom which attracts [the probe].
\end{abstract}

I: So you do feel something where the balls [of the displayed ball-and-stick representation] are, then?

S2: Well yes, at the oxygen atoms I do.

\section{(32:41-33:08 min.)}

Although S2 was using the haptic force-and-follow mode, the student was specifically informed earlier in the interview that the model does not provide any force feedback in the vicinity of the atom centres. Instead, it seems that this student's judgment is based primarily on an expectation of what any experienced force ought to feel like at that position.

The datum exposed by S2 suggests that students' conceptual knowledge did not always map correctly to the scientific concepts underlying the virtual environment. That is, students sometimes interpreted their visuohaptic experiences in terms of chemical ideas that the virtual system was not designed to model. As a result, in some cases, students expressed ideas about chemical concepts that were scientifically inaccurate (e.g., Figure 4). The data also suggested that not all conceptual engagement was related to the interactive experience garnered from the virtual environment. Similarly, some of the students' verbalisations during interacting with the model were pure descriptions of their multimodal experiences of using the system per se.

\subsection{Concluding Discussion of the Findings and Limitations of} the Study. This study has shown that prior to exposure to the visuohaptic model, students' written responses displayed that they struggled to distinguish between the concept of polarity and electric field, which may have resulted in the unscientific view that all nonpolar molecules are void of an electric field. A potential source of this erroneous reasoning would be due to descriptions of nonpolarity that emphasise a "cancellation of charges." One way to potentially remedy this scenario would be to directly engage electric field concepts into teaching polarity and intermolecular interactions. Also, rather than depicting intermolecular forces as separate definition "boxes," emphasis should be given to properties common to all electrostatic interactions (e.g., [20]).

The case-based interactive data analysed in this study have indicated that tactile interaction may induce an active integration of electric field knowledge with molecular charge distribution. In this regard, the visuohaptic interaction with the dynamic bimodality offered by the environment may foster the development of electric field concepts (cf. [3]). In doing so, the multisensory visuohaptic experience of molecular properties promotes the fusion of physical and chemical concepts that are otherwise taught and treated as isolated pieces of knowledge.

The findings of this study contribute to the educational research literature by presenting case-based descriptions of some nuances of the actual process during which learning may be occurring in the presently described multisensory 
context. Although we have intriguing insight into how learners may be interacting with features of the visuohaptic environment in processing molecular electric fields, we also acknowledge that other work on visuohaptic platforms has shown little or no gain in comparison with visual-only environments. Given that our study did not specifically seek to investigate such differences, it is interesting to acknowledge that Minogue et al. [9] found only small learning differences after students' interaction with a haptic model of a cell, while Wiebe et al. [8] demonstrated that interaction with a visuohaptic simulation that represented principles of levers did not provide an improved understanding. We suggest that the educational benefits of visuohaptic environments are closely related to the intended conceptual learning that is envisaged, as supported in the context under study here, where actually experiencing modelled electrostatic forces are very much part-and-parcel of the conceptual understanding targeted for attainment. Albeit so, it remains unexplored as to what extent a visual-only system of electrostatic molecular phenomena would deliver a similar type of processing and conceptual impact as that observed here.

Another potential shortcoming of the study is that the qualitative case-based and small-scale nature of the study does not allow any statistical generalisations to be garnered based on the findings although we wish to maintain our position that we purposefully solicited a description of the nature of interacting with the virtual environments, rather than on inferring any large-scale generalisations at this stage of the project. Lastly, although learning gains in the form of improved test scores certainly serve as one type of validation of the effectiveness of a particular educational intervention, the focus of the current case-based research is motivated by the assumption that the design of effective multisensory learning platforms first requires deeper insight into the actual process for why learning may (or may not) be occurring (e.g., [7]).

\section{Implications of the Findings for Developing Multisensory Virtual Nanoenvironments}

Given the broader aims of the research thrust reported in the current paper, the case-based findings can be directly applied to a research-based approach for the development of similar systems that represent nanorelated ideas. We present these applications as the following three perspectives.

5.1. Conceptual Perspective. The results delivered in this paper suggest that the model can be further developed to convey multiple concepts related to nanoscientific knowledge and properties. For example, conceptual aspects of the "big ideas" of nanoscale principles captured by Stevens et al. [20] and Wansom et al. [41] can be brought directly to bear upon interaction with this virtual environment, even in its current state. For example the representation of the distribution of electrostatic charges and multiple charge-charge interactions rendered in the model are directly related to the core nanoscale idea of forces and interactions. The rendering of the field lines and haptic interaction offered through the use of the stylus-tip to "feel" the electrostatic forces around the molecule conveys a conceptualisation of the shape and topography of nanoscale entities. Furthermore, multiple visual representations of different structural and volumetric aspects of a molecule such as van der Waals radii and electrostatic field lines provide insight into the relative size and scale of these properties within the same nanoobject. Lastly, by receiving a multisensory experience that simultaneously couples visual and haptic perception could serve as a powerful means for enhancing conceptual understanding of phenomena beyond our direct observation.

Based on these conceptual inroads, future development of the model will be directed to representing other overarching nanoproperties such as the constant random motion of nanoobjects in colloidal systems and nanophysical properties such as the extraordinarily high length-to-diameter and surface-to-volume ratios that nanoobjects exhibit. Doing so will allow learners to create conceptual linkages between these nanofeatures and the often counterintuitive and unexpected size-dependent properties that nanoobjects exhibit at the nanoscale.

5.2. Interactive Perspective. The presented findings also shape intriguing inroads into how to think about the nature of human-system interaction when it comes to developing such virtual models. For one, this study provides clear case-based evidence that there are different styles and strategies of interaction that a learner may employ when immersed in a virtual environment that aims to deliver science concepts (Table 1). On this score, it is interesting to observe from the data presented here that while one student may carry out their virtual explorations with clear expectations of what the experience may "confirm," another may be much more "explorative" in the way they engage their interaction. Hence, the data presented here provides rich evidence that educational role players should not merely assume that learners will interact with virtual models in the ways automatically expected or intended.

As has also been demonstrated through cases represented within the emergent $2 \times 2$ intersection of the conceptual knowledge and emergent interactive strategy dimensions (Table 1), it is crucial to monitor and reflect upon how students connect the interactive experience with the conceptual domain intended to be communicated. With respect to designing virtual nanotechnology environments, we will apply these results to focus on considering what nature of interaction is afforded in such contexts relative to the conceptual knowledge intended to be conveyed. For instance, utilisation of appropriate interactive cues for evoking certain conceptual knowledge engagement, or for stimulating new knowledge construction, will be sought in parallel with appropriate modes of multisensory interaction (e.g., investigating various gestural hand outputs) that could best support such knowledge acquirement.

5.3. Design Perspective. The results also provide inroads into informing the design of such environments when it comes to the desire to portray nanophenomena. The data presented here shows that there is clearly an intimate alliance that 
exists between the learner, the actual virtual model, and the nature of the interaction that the learner exhibits. Thus, the case-based data revealed in this study suggests that the development of future environments will be shaped by considering an indispensable union between four actors, namely, the nano-concepts to be communicated, the nature of the user tasks that are intended to afford a conceptualisation of these nano-concepts, the interactive features offered by the system, and the extrinsic design features of the system.

As supported by the results described in this paper, we are of the view that any conceptual gain and insight that is provided from engaging with visuohaptic virtual environments such as that exposed here is due to the user's real-time interactive experience of performing the task of interest. In this way, we hypothesise that it is the specific bimodal behavioural experience afforded by the task that is the cognitive vehicle for engendering the conceptual knowledge underlying the virtual reality representation. Since the time of writing, we have commenced with application of aspects of the findings reported here into the development of multisensory virtual architectures for communicating core nanoconcepts and nanoproperties to learners. Eventual application, implementation, and potential benefit of components of these virtual architectures in real classroom settings remain the long-term pedagogical quest of the broader research vision.

\section{Acknowledgments}

Grants 2008-5077 and 2011-5569 from the Swedish Research Council (Vetenskapsrådet) supported this paper. The authors kindly thank the student volunteers for participating in the data collection.

\section{References}

[1] E. Richard, A. Tijou, P. Richard, and J. L. Ferrier, "Multi-modal virtual environments for education with haptic and olfactory feedback," Virtual Reality, vol. 10, no. 3-4, pp. 207-225, 2006.

[2] S. J. Lederman and R. L. Klatzky, "Hand movements: a window into haptic object recognition," Cognitive Psychology, vol. 19, no. 3, pp. 342-368, 1987.

[3] M. Reiner, "Conceptual construction of fields through tactile interface," Interactive Learning Environments, vol. 7, no. 1, pp. 31-55, 1999.

[4] J. Minogue and M. G. Jones, "Haptics in education: exploring an untapped sensory modality," Review of Educational Research, vol. 76, no. 3, pp. 317-348, 2006.

[5] K. J. Schönborn, P. Bivall, and L. A. E. Tibell, "Exploring relationships between students' interaction and learning with a haptic virtual biomolecular model," Computers and Education, vol. 57, no. 3, pp. 2095-2105, 2011.

[6] M. Wilson, "Six views of embodied cognition," Psychonomic Bulletin and Review, vol. 9, no. 4, pp. 625-636, 2002.

[7] C. Dede, "Immersive interfaces for engagement and learning," Science, vol. 323, no. 5910, pp. 66-69, 2009.

[8] E. N. Wiebe, J. Minogue, M. G. Jones, J. Cowley, and D. Krebs, "Haptic feedback and students' learning about levers: unraveling the effect of simulated touch," Computers and Education, vol. 53, no. 3, pp. 667-676, 2009.
[9] J. Minogue, M. G. Jones, B. Broadwell, and T. Oppewall, “The impact of haptic augmentation on middle school students' conceptions of the animal cell," Virtual Reality, vol. 10, no. 3-4, pp. 293-305, 2006.

[10] C. Furió and J. Guisasola, "Difficulties in learning the concept of electric field," Science Education, vol. 82, no. 4, pp. 511-526, 1998.

[11] M. C. Pocoví, "The effects of a history-based instructional material on the students' understanding of field lines," Journal of Research in Science Teaching, vol. 44, no. 1, pp. 107-132, 2007.

[12] S. Törnkvist, K.-A. Petterson, and G. Tranströmer, "Confusion by representation: on students comprehension of the electric field concept," American Journal of Physics, vol. 61, no. 4, pp. 335-338, 1993 .

[13] D. Clark and D. Jorde, "Helping students revise disruptive experientially supported ideas about thermodynamics: computer visualizations and tactile models," Journal of Research in Science Teaching, vol. 41, no. 1, pp. 1-23, 2004.

[14] C. Dede, M. Salzman, R. B. Loftin, and K. Ash, "The design of immersive virtual learning environments: fostering deep understandings of complex scientific knowledge," in Innovations in Science and Mathematics Education: advanced Designs for Technologies of Learning, M. Jacobson and R. B. Kozma, Eds., pp. 361-414, Erlbaum, Mahwah, NJ, USA, 2000.

[15] C. Dede, M. C. Salzman, and R. B. Loftin, "ScienceSpace: research on using virtual reality to enhance science education," in Proceedings of the ED-MEDIA Conference (AACE '96), $\mathrm{P}$. Carlson and F. Makedon, Eds., pp. 172-177, Charlottesville, Va, USA, 1996.

[16] A. Křenek, "Haptic rendering of molecular conformations," in Proceedings of the EuroHaptics 2001, C. Baber, M. Faint, S. Wall, and A. Wing, Eds., Educational Technology Research Paper Series, pp. 142-145, University of Birmingham, Birmingham, UK, July 2001.

[17] K. L. Palmerius, M. Cooper, and A. Ynnerman, "Haptic rendering of dynamic volumetric data," IEEE Transactions on Visualization and Computer Graphics, vol. 14, no. 2, pp. 263-276, 2008.

[18] T. R. Tretter, M. G. Jones, T. Andre, A. Negishi, and J. Minogue, "Conceptual boundaries and distances: students' and experts' concepts of the scale of scientific phenomena," Journal of Research in Science Teaching, vol. 43, no. 3, pp. 282-319, 2006.

[19] A. M. Stoneham, "The challenges of nanostructures for theory," Materials Science and Engineering C, vol. 23, no. 1-2, pp. 235-241, 2003.

[20] S. Y. Stevens, L. M. Sutherland, and J. S. Krajcik, The Big Ideas of Nanoscale Science and Engineering: A Guidebook for Secondary Teachers, NSTA Press, Arlington, Va, USA, 2009.

[21] B. Hingant and V. Albe, "Nanosciences and nanotechnologies learning and teaching in secondary education: a review of literature," Studies in Science Education, vol. 46, no. 2, pp. 121152, 2010.

[22] C. A. Batt, A. M. Waldron, and N. Broadwater, "Numbers, scale and symbols: the public understanding of nanotechnology," Journal of Nanoparticle Research, vol. 10, no. 7, pp. 1141-1148, 2008.

[23] K. M. Eisenhardt, "Building theories from case study research," Academy of Management Review, vol. 14, no. 4, pp. 532-550, 1989.

[24] V. Dawson, "An exploration of high school (12-17 year old) students' understandings of, and attitudes towards biotechnology 
processes," Research in Science Education, vol. 37, no. 1, pp. 5973, 2007.

[25] A. G. Harrison, D. J. Grayson, and D. F. Treagust, "Investigating a grade 11 student's evolving conceptions of heat and temperature," Journal of Research in Science Teaching, vol. 36, no. 1, pp. 55-87, 1999.

[26] A. G. Harrison and D. F. Treagust, "Learning about atoms, molecules, and chemical bonds: a case study of multiple-model use in grade 11 chemistry," Science Education, vol. 84, no. 3, pp. 352-381, 2000.

[27] S. B. Merriam, Qualitative Research and Case Study Applications in Education, Jossey-Bass, San Francisco, Calif, USA, 1998.

[28] F. Erickson, "Qualitative methods in research on teaching," in Handbook of Research on Teaching, M. Wittrock, Ed., pp. 119161, Macmillan, New York, NY, USA, 3rd edition, 1986.

[29] K. J. Schönborn and T. R. Anderson, "A model of factors determining students' ability to interpret external representations in biochemistry," International Journal of Science Education, vol. 31, no. 2, pp. 193-232, 2009.

[30] C. Y. Tsui and D. F. Treagust, "Understanding genetics: analysis of secondary students' conceptual status," Journal of Research in Science Teaching, vol. 44, no. 2, pp. 205-235, 2007.

[31] R. K. Yin, Case Study Research: Design and Methods, Sage, Thousand Oaks, Calif, USA, 1994.

[32] B. G. Glaser and A. L. Strauss, The Discovery of Grounded Theory, Aldine, Chicago, Ill, USA, 1967.

[33] Y. S. Lincoln and E. G. Guba, Naturalistic Inquiry, Sage, Newbury Park, Calif, USA, 1985.

[34] H. K. Wu, J. S. Krajcik, and E. Soloway, "Promoting understanding of chemical representations: students' use of a visualization tool in the classroom," Journal of Research in Science Teaching, vol. 38, no. 7, pp. 821-842, 2001.

[35] M. D. Gall, W. R. Borg, and J. P. Gall, Educational Research: An Introduction, Longman, New York, NY, USA, 6th edition, 1996.

[36] R. Justi and J. van Driel, "A case study of the development of a beginning chemistry teacher's knowledge about models and modelling," Research in Science Education, vol. 35, no. 2-3, pp. 197-219, 2005.

[37] S. Eigner-Thiel and S. Bögeholz, "Bildung für Nachhaltige Entwicklung aus Sicht von MultiplikatorInnen außerschulischer Bildungsträger," Umweltpsychologie, vol. 8, no. 2, pp. 80100, 2004.

[38] P. J. Russell, S. L. Wolfe, P. E. Hertz, C. Starr, and B. McMillan, Biology: The Dynamic Science, Thomson, Belmont, Calif, USA, 2008.

[39] A. Baddeley, "Working memory," Science, vol. 255, no. 5044, pp. 556-559, 1992.

[40] E. von Glasersfeld, “Cognition, construction of knowledge, and teaching," Synthese, vol. 80, no. 1, pp. 121-140, 1989.

[41] S. Wansom, T. O. Mason, M. C. Hersam et al., "A Rubric for post-secondary degree programs in nanoscience and nanotechnology," International Journal of Engineering Education, vol. 25, no. 3, pp. 615-627, 2009. 

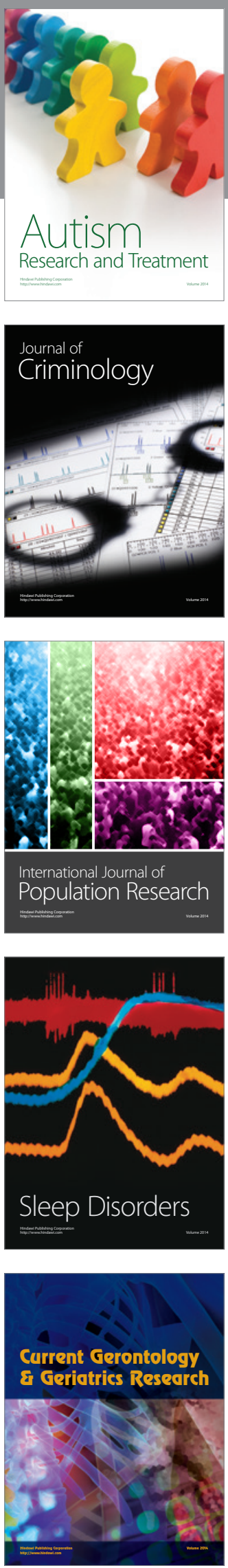
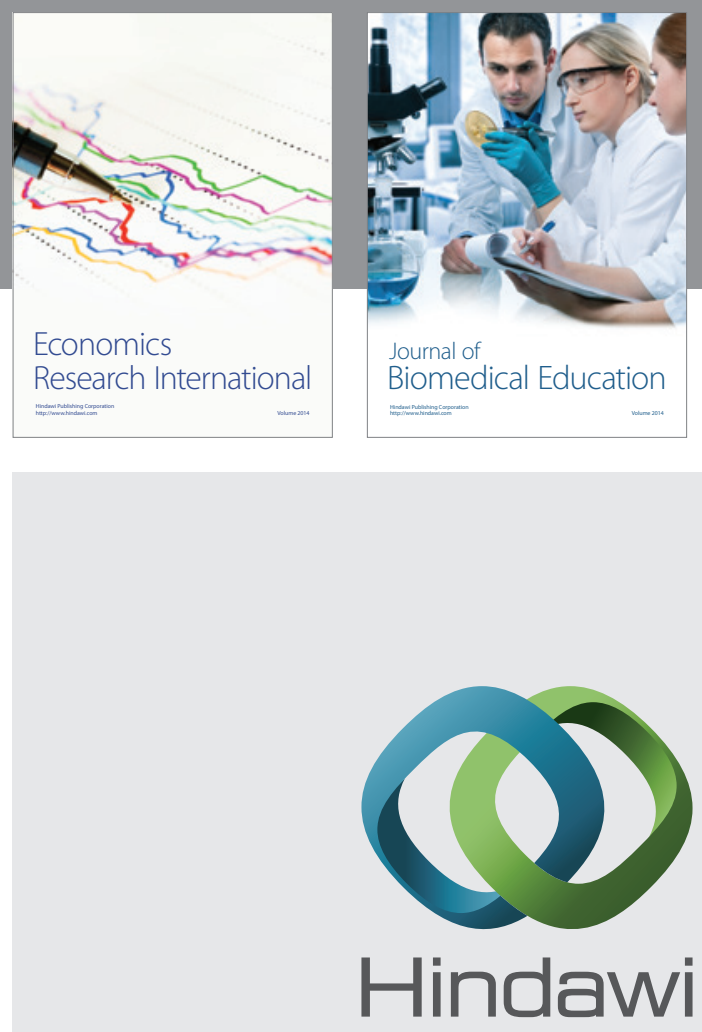

Submit your manuscripts at

http://www.hindawi.com
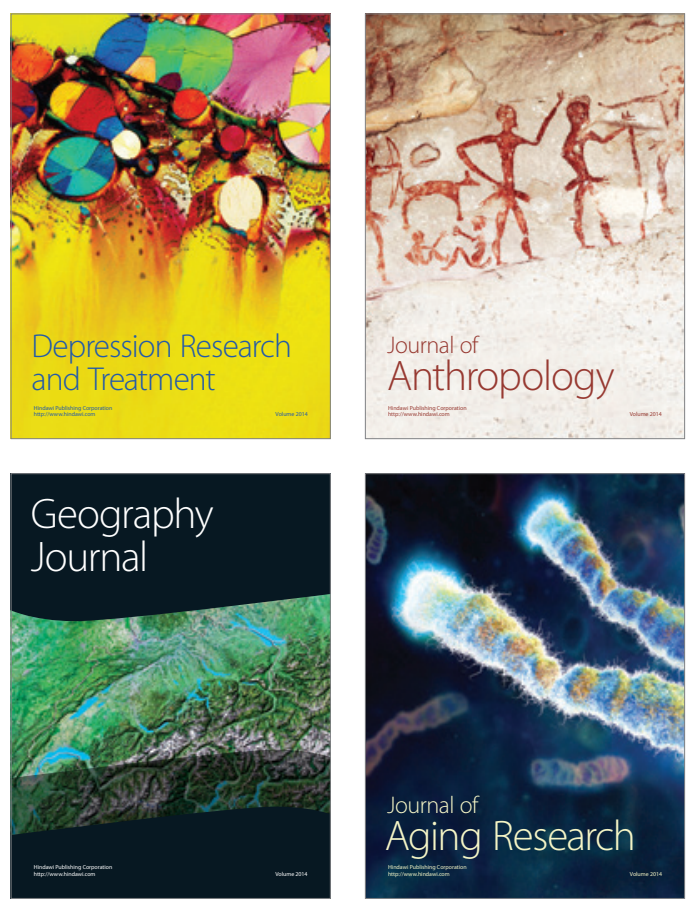
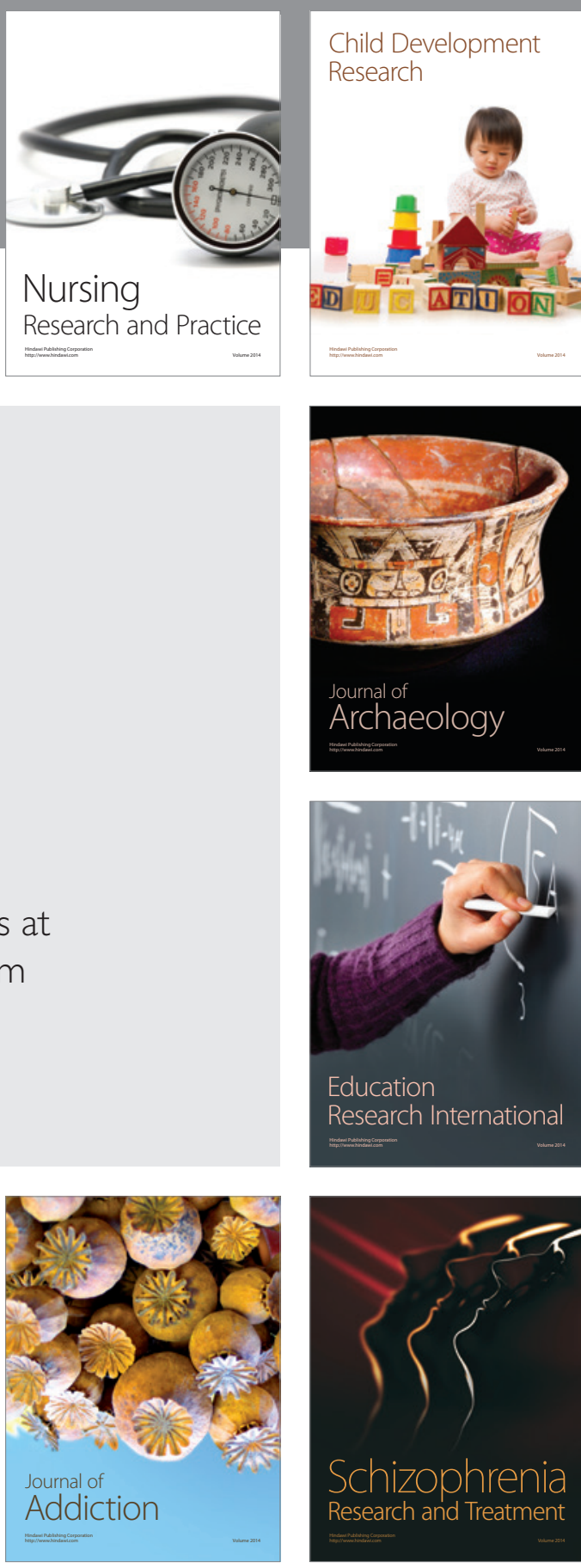

(D)
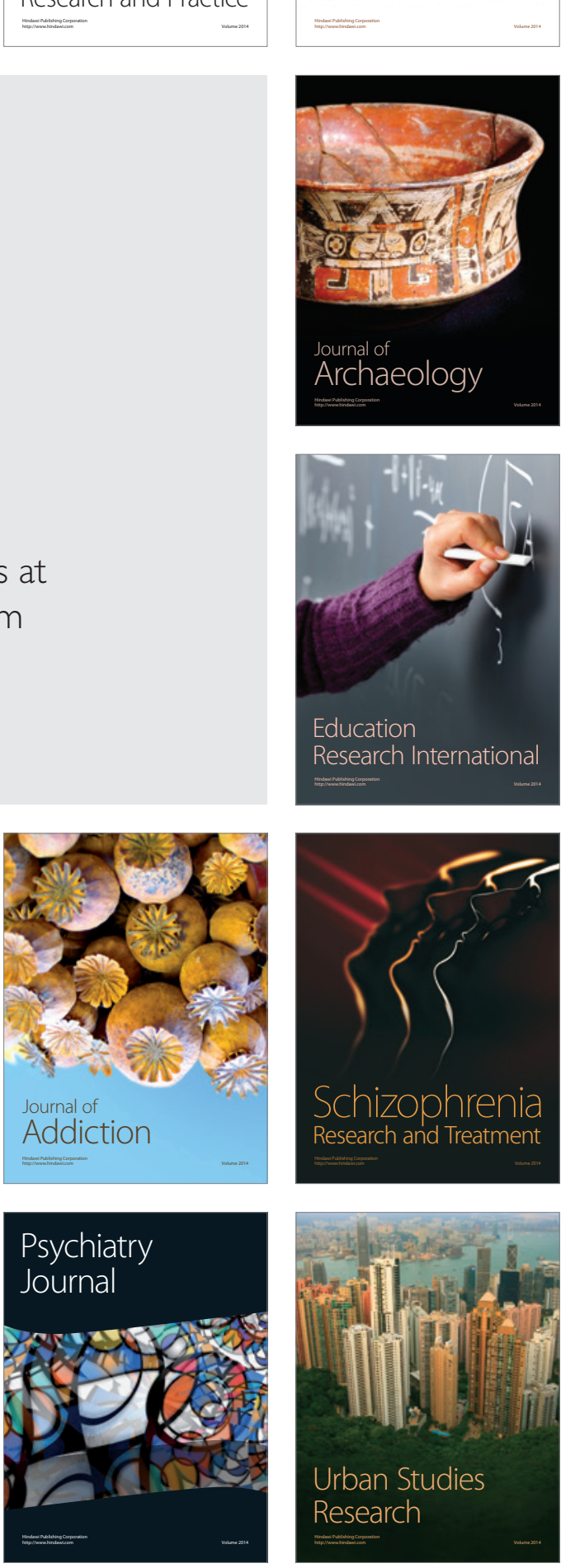